\title{
International Journal of Dentistry and Oral Health
}

\section{Treatment Regime for Crown Fractures: Autogenous Tooth Fragment Reattachment - Case Reports}

\author{
Nagaveni NB*, Bajaj M, Lobo $\mathbf{N}$ and Poornima $\mathbf{P}$ \\ Department of Pedodontics and Preventive Dentistry, College of Dental Sciences, Davangere, \\ Karnataka, India
}

${ }^{*}$ Corresponding author: Dr. N.B. Nagaveni, Reader, Department of Pedodontics and Preventive Dentistry, College of Dental Sciences, Davangere, Karnataka, India, E-mail: nagavenianurag@ gmail.com

\section{Introduction}

Traumatic dental injuries are a common occurrence in routine dental practice. These injuries in addition to the amount of physical pain are notorious for the psychological impact on the patient. Anterior tooth fractures are frequently seen and a high prevalence has been noted in the age group of 7-12 years [1]. Fracture of coronal portion of tooth is often seen in children and adolescents. Crown fractures of permanent incisors account for $18-22 \%$ of all dental traumas among which $96 \%$ involve maxillary central incisors [2].

The management of coronal tooth fracturesis dependent on many factors such as biological width violation, endodontic involvement, and pattern of fracture, presence or absence of fractured fragment, restorability of tooth, occlusion and aesthetics [3]. Many techniques such as stainless steel crowns, orthodontic bands, ceramic crowns and composite resin restorations have been used in the management of such fractures [4]. Despite successful outcomes, drawbacks such as sacrifice of healthy tooth structure and compromised esthetics has compelled us to search and reviewother alternativetreatment modalities.

Tooth fragment reattachment is a technique by which fractured fragment is reattached using adhesive cement. This method is preferred when the fractured fragment is available as it offers the advantage of use in an emergency esthetic situation. It is considered a favorable alternative to conventional techniques since it doesn't require additional tooth preparation, requires less time, provides excellent esthetics and has the psychological impact of having used the natural tooth fragment for restoration of esthetics.

This article presents a case series of management of uncomplicated and complicated crown fractures using tooth fragment reattachment technique.

\section{Case Report 1}

A 13 year old female patient reported to the Department of Pedodontics and Preventive Dentistry, College of Dental Sciences, Davangere , Karnataka with the chief complaint of broken upper front teeth following trauma due to fall in classroom half an hour prior. She had brought the broken fragments stored in milk.

Clinical examination revealed an Ellis Class II fracture with 11. The left maxillary incisor showed an Ellis Class III fracture in 21 with pin point exposure of pulp. The whole fragment of 21 was available while 2 fragments of 11 were available (Figure 1).

A periapical radiographic examination revealed an oblique fracture of 11 involving enamel and dentin and oblique fracture of 21 involving enamel, dentin and pulp. No root resorption was evident in the radiographs. Since the patient had brought the fractured fragments, it elicited a desire by the patient to restore the natural tooth. Hence natural tooth fragment reattachment procedure was sought as the method for managing the condition.

When the patient presented to the department, direct pulp capping was performed on 21 with aid of calcium hydroxide and GIC was applied on both the incisors after isolation. The fractured fragments were stored in saline (Figure 2a). The next day reattachment procedure was conducted. In 11 , since the fragment was in 2 parts a bevel was placed prior to bonding (Figure 3a). The fractured fragments were held together using composite restoration (Figure $2 \mathrm{~b}$ ). Both the tooth as well as the fractured fragment were etched and bonded prior to reattachment. Favorable results were obtained at periodic recalls of 1, 3 and 6 months (Figure $3 b$ ).

\section{Case Report 2}

A 12 year old male patient reported to the same department with the chief complaint of pain and broken upper front tooth following trauma while play 4 days ago. He had brought the broken fragments stored in milk.

Clinical examination revealed an Ellis Class III fracture of the right maxillary incisor (Figure 4). A periapical radiograph examinationrevealed an oblique fracture of 11 involving enamel and dentin and pulp. Since the parent had expressed an interest in use of the fractured fragment to restore the tooth, fragment reattachment procedure was decided as the treatment regimen for management of this case.

Root canal therapy for 11 was suggested. The fractured fragment was stored in saline (Figure 5). After root canal treatment; discoloration was

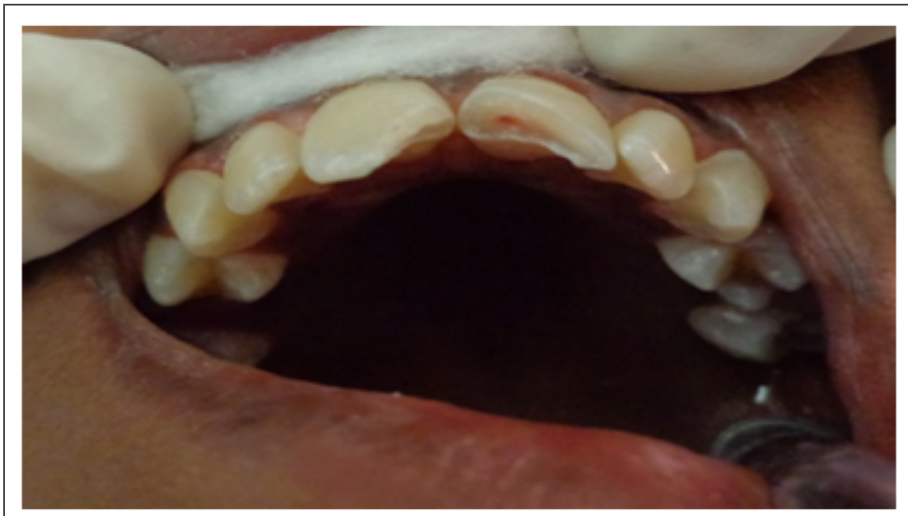

Figure 1: Intraoral view showing fractured maxillary incisors. Ellis Class I in 11 and Ellis Class III in 21 with pin point pulp exposure 


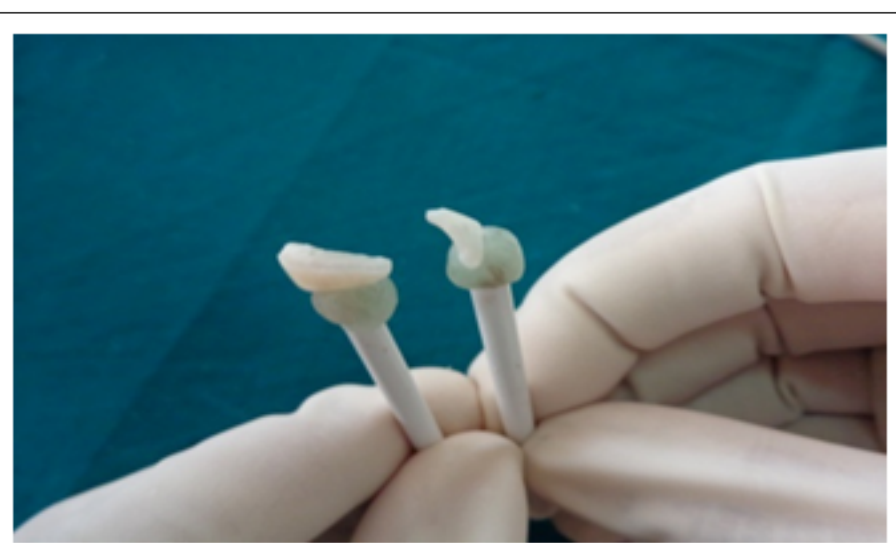

Figure 2a: Fractured fragments bevel in 11 and simple

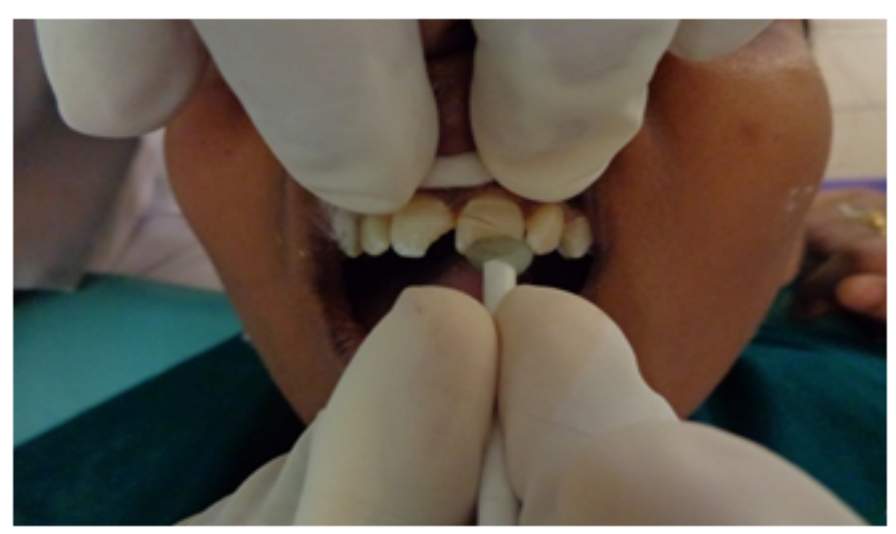

Figure $2 \mathbf{b}$ : Joining of the 2 fragments and built up with 21 composite for attachment in 11 months recall

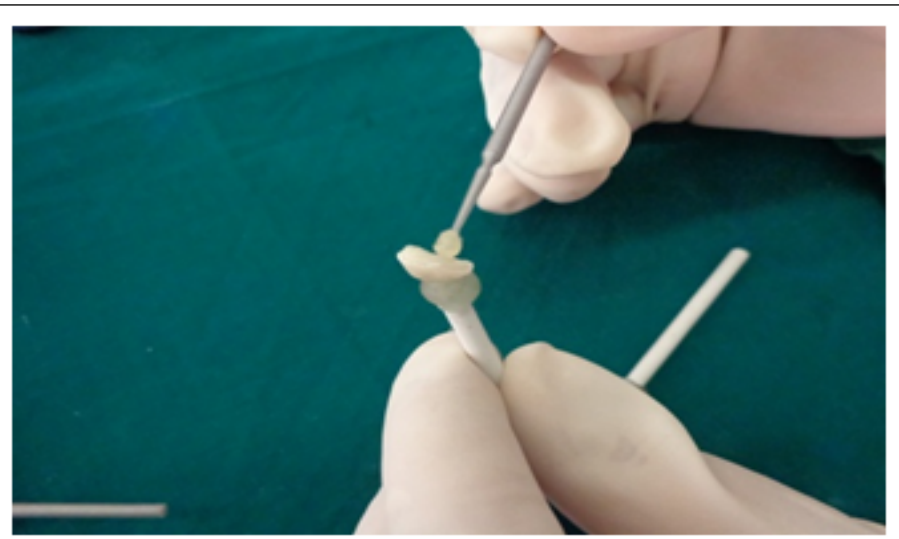

Figure 3a: Placement of bevel in 11 and simple reattachment in 21

observed and treated by intracoronal bleaching using sodium perborate (Figure 6a). Fragment reattachment was done a month later after completion of root canal treatment and intracoronal bleaching (Figure $6 \mathrm{~b})$. In this case, bevel was placed prior to bonding of the fragment. The surface of tooth and fractured fragment was etched and bonded prior to reattachment. Favorable results were obtained at periodic recalls of 1,3 and 6 months (Figures $7 \mathrm{a}$ and $7 \mathrm{~b}$ ).

\section{Discussion}

Fracture of anterior teeth after trauma is not only painful to the patient but also affects the psychological well-being of the patient.It is advisable

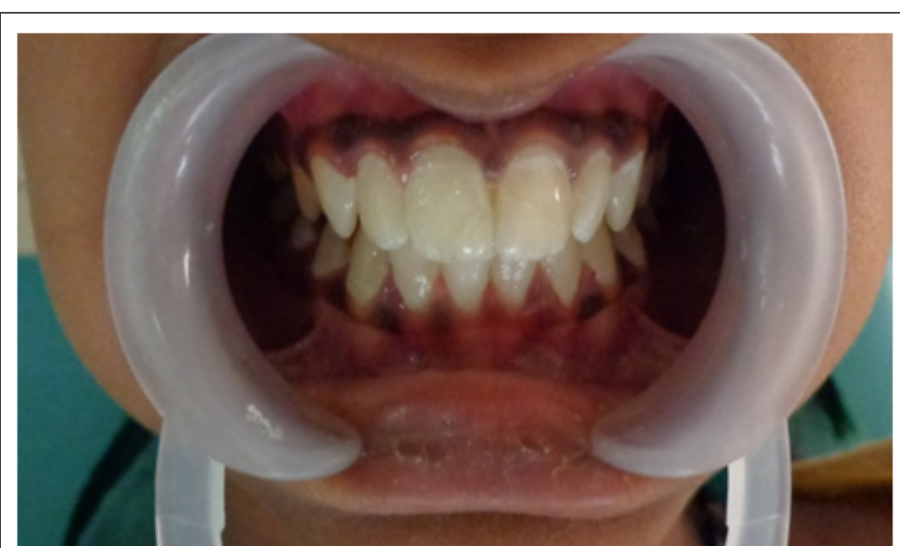

Figure 3b: Post operative view at 6 months recall

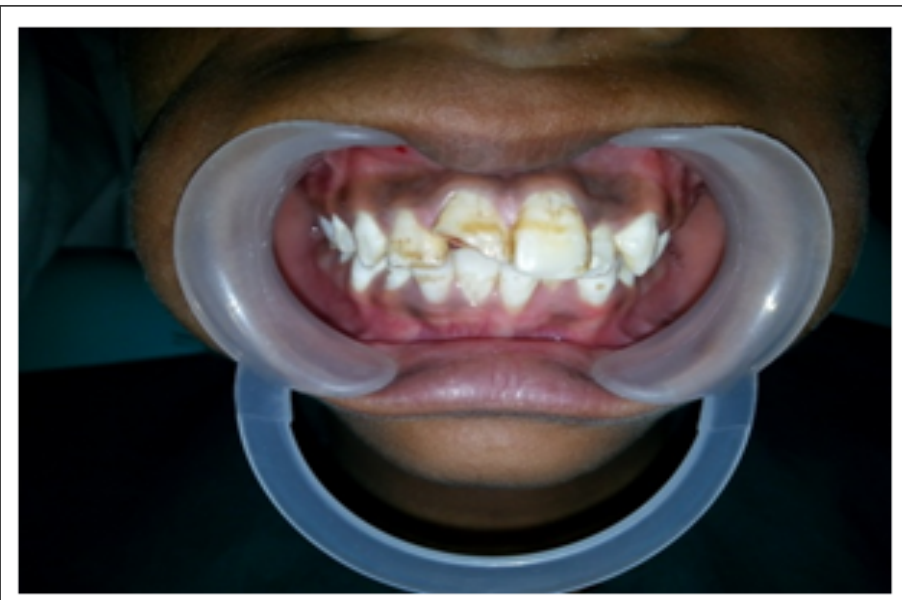

Figure 4: Pre operative view of Ellis Class III in 11

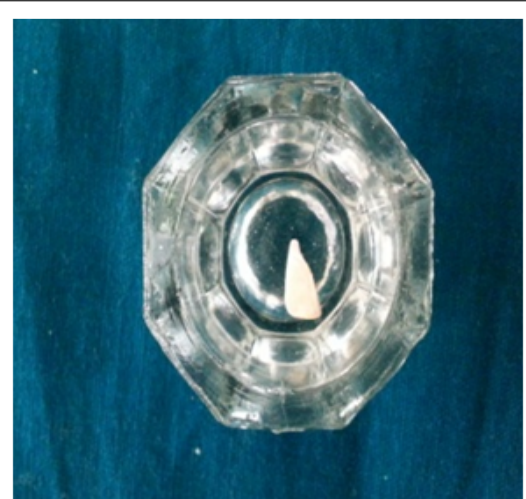

Figure 5: Fractured fragment stored in saline

that whenever the fracture fragment is available reattachment should be the first choice of treatment [5]. The use of natural tooth fragment to restore the fractured tooth offers conservative, esthetic, and economical advantages. It ensures long lasting esthetics and is a simple procedure. It retains the translucency of natural tooth and its abrasive resistance is better than composites. Several studies have shown that the impact strength of reattached tooth is not significantly different from that of intact natural tooth [6]. Fragment reattachment technique has some disadvantages such as lesser than ideal esthetics (caused by dehydration of tooth), unknown longevity and chances of separation of the repair caused by progressive breakdown of the bonded junction [7]. Hence continuous monitoring

Citation: Nagaveni NB, Bajaj M, Lobo N, and Poornima P (2015) Treatment Regime for Crown Fractures: Autogenous Tooth Fragment Reattachment Case Reports. Int J Dent Oral Health 1(6): doi http://dx.doi.org/10.16966/2378-7090.136 


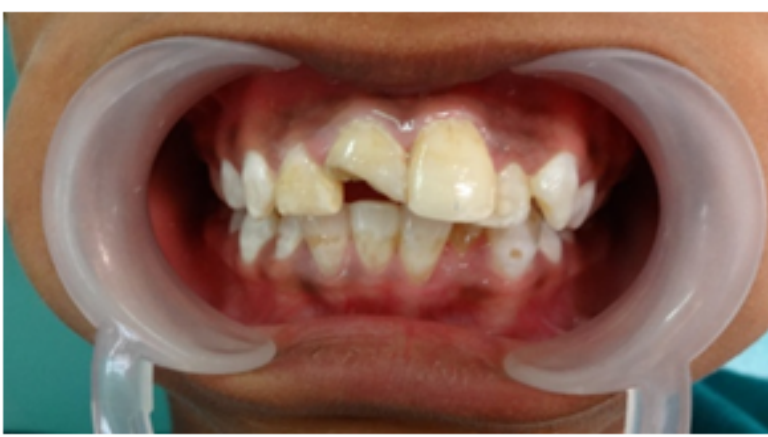

Figure 6a: After intra coronal bleaching

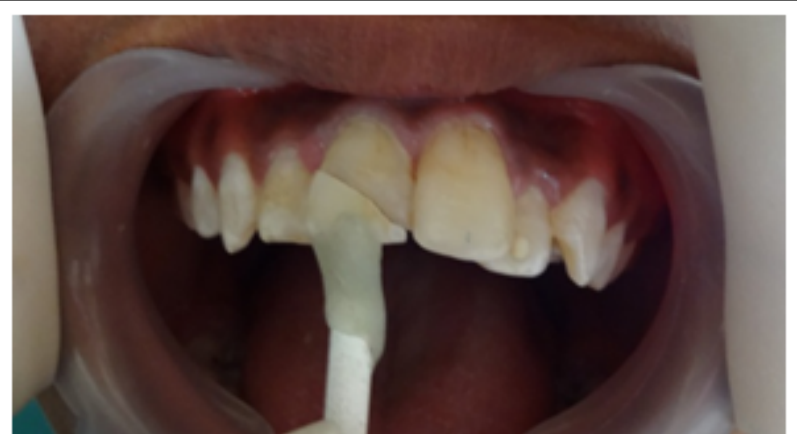

Figure 6b: Fragment reattachment

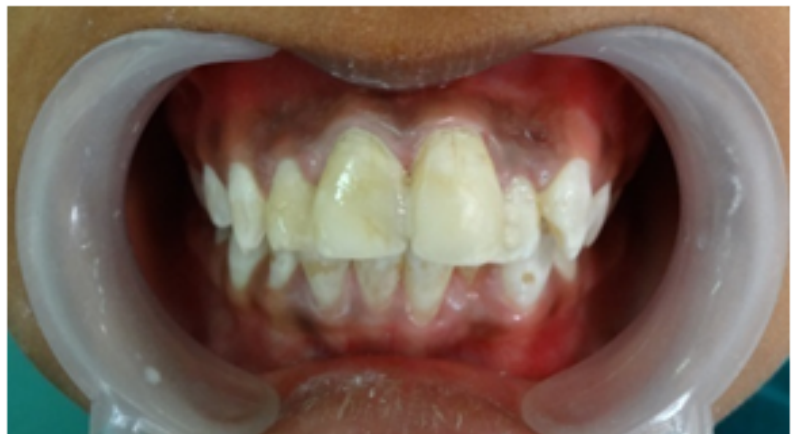

Figure 7a: Post operative view at 6 months recall

is necessary. Use of newer materials in addition to strict adherence to treatment protocol and isolation during bonding procedures can ensure good aesthetics with good prognosis of the restored fractured fragment. Reattachment procedure is often multidisciplinary and depends on the extension of tooth fracture and injury to the attachment apparatus. Since both cases were supragingival fractures, gingivectomy was not required in our cases. Reattachment failures can occur as a result of new trauma or parafunctional habits, so patient education about treatment limitations can enhance clinical success [8].

Various techniques and designs have been proposed for reattachments of fractured tooth fragments, like simple reattachment, enamel beveling, $\mathrm{V}$ shaped internal enamel groove, internal dentin groove, external chamfer and overcontour $[9,10]$.

Reis et al concluded that a simple reattachment with no further preparation of the fragment or tooth could restore only $37.1 \%$ of intact tooth's fracture resistance, but that of buccal chamfer recovered $60.6 \%$ of that fracture resistance and bonding, with an over contour and placement of an internal groove restores fracture strengths of $97.2 \%$ and $90.5 \%$ respectively [9]. In the first case since the fragment was broken in two

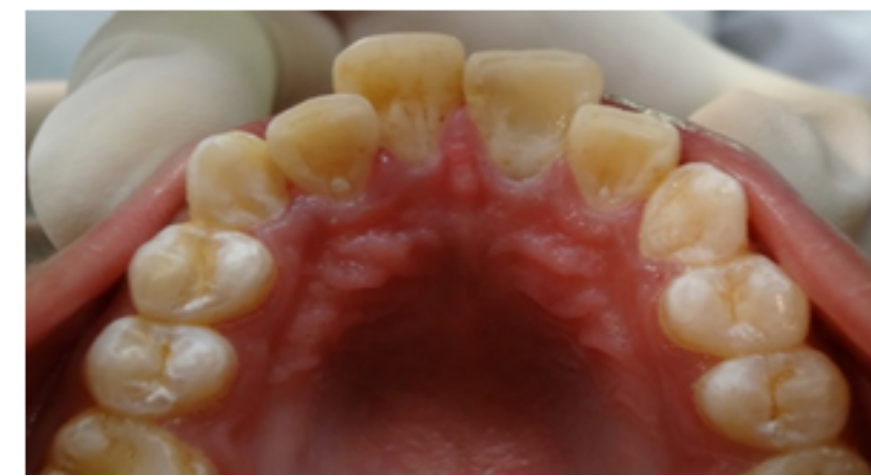

Figure 7b: Post operative intra oral view at 6 months recall

pieces, an additional bevel preparation was suggested to ensure successful bonding and longevity in the long run. With all traumatic injuries, follow up is of critical importance and the patient should be followed for 3,6 and 12 months and yearly for 5 years. At these follow-up visits esthetics, tooth mobility and periodontal status should be confirmed both clinically and radio graphically.

\section{Conclusion}

Since fragment reattachment technique guarantees many advantages it is essential to spread awareness to ensure that fragments are preserved during incidences of trauma although initial results of fragment reattachment are encouraging, a long term follow up is required to confirm the periodontal stability of the affected teeth.Hence detailed record of follow up is warranted in all cases of fragment reattachment.

\section{References}

1. Kulkarni VK, Bhusari CP, Sharma DS, Bhusari P, Bansal AV, et al. (2014) Autogenous tooth fragment reattachment: A multidisciplinary management for complicated crown-root fracture with biologic width violation. J Indian Soc Pedod Prev Dent 32: 190-4.

2. Uddin MF, Nasser M, Howlander MMR, Alam MS, Nabi MN (2012) Changing concepts in reattachment of tooth fracture. Update Dent Coll J 2: 42-6.

3. Macedo GV, Diaz PI, De O Fernandes CA, Ritter A (2008) Reattachment of anterior teeth fragments: A conservative approach. J Esthet Restor Dent 20: 5-18.

4. Buonocore MG, Davila J (1973) Restoration of fractured anterior teeth with ultraviolet light polymerized bonding materials: a new technique. J Am Dent Assoc 86: 1349-4.

5. Belchema A (2008) Reattachment of fractured permanent incisors in school children (review). J IMAB 14: 96-9.

6. Farik B, Munksgaard EC, Andreasen JO (2000) Impact strength of teeth restored by fragment bonding. Dent Traumatol 16: 151-3.

7. AbdulkhayumA, Munjal S, Babaji $P$, Chaurasia VR, Munjal $S$, et al. (2014) In vitro evaluation of fractured strength recovery of reattached anterior fractured tooth fragment using different re-attachment techniques. J Clin Diagn Res 8: 208-1.

8. Andreasen FM, Noren JG, Andreasen JO, Engelhardtsen S, LindhStromberg U (1995) Long term survival of fragment bonding in the treatment of fractured crowns: a multicenter clinical study. Quintessence Int 26: 669-1.

9. Reis A, Kraul A, Francci C, de Assis TG, Crivelli DD, et al. (2001) Reattachment of anterior fractured teeth: Fracture strength using different technique. Ope Dent 26: 287-284.

10. Nagaveni NB, Pathak S, Poornima P, Roshan NM (2014) A judicious restorative approach for management of fractured anterior teeth : Report of three cases. Niger J Exp Clin Diorci 2: 125-9. 